\title{
High Spatiotemporal Resolution Observation of Glutathione Hydropersulfides in Living Cells and Tissue via a Two-Photon Ratiometric Fluorescent Probe
}

\author{
Yue Wang, ${ }^{\dagger, \perp}$ Liangwei Zhang, ${ }^{\dagger}$ Songzi Zhang, ${ }^{\dagger}$ Zhe Liu, ${ }^{\S}$ and Lingxin Chen ${ }^{*}, \dagger, \$, \|_{\odot}$ \\ ${ }^{\dagger}$ CAS Key Laboratory of Coastal Environmental Processes and Ecological Remediation, Yantai Institute of Coastal Zone Research, \\ Chinese Academy of Sciences, Yantai 264003, China \\ ${ }^{\ddagger}$ Laboratory for Marine Biology and Biotechnology, Pilot National Laboratory for Marine Science and Technology, Qingdao \\ 266237, China \\ ${ }^{\S}$ Department of Chemistry and Chemical Engineering, Qufu Normal University, Qufu, 273165, China \\ "Center for Ocean Mega-Science, Chinese Academy of Sciences, 7 Nanhai Road, Qingdao 266071, China \\ ${ }^{\perp}$ University of Chinese Academy of Sciences, Beijing 100049, China
}

Supporting Information

ABSTRACT: Glutathione hydropersulfides (GSSH) are alluded to play crucial roles in signal transduction, redox homeostasis, and metabolic regulation. However, the detailed biological functions of GSSH in these aspects are extremely ambiguous. The key barrier to understand the role of GSSH in biological systems is a lack of detection tools with high spatiotemporal resolution. To address the issues, we are seeking novel chemical tools for GSSH detection. We herein develop the first two-photon ratiometric fluorescent probe (TP-Dise) for GSSH detection with high spatial and temporal resolution in living cells and

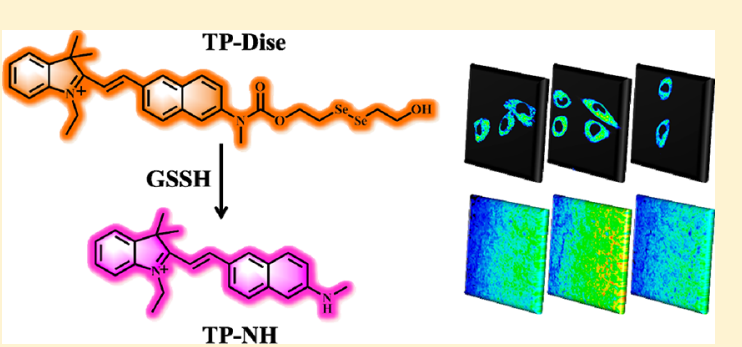
tissue. On the basis of our probe TP-Dise, we investigate the biosynthesis of GSSH, and the results indicate that GSSH is mainly from two sulfurtransferases, CBS and CSE. Furthermore, we explore the biological function of GSSH in protecting cells from mercury ion-induced cell damage for the first time. The experimental results indicate that mercury ions may induce cell death by causing mitochondrial autophagy. GSSH acts both as antagonist and as antioxidant and can effectively alleviate the damage caused by mercury stress.

$\mathrm{H}$ ydropersulfides (RSSH), a kind of reactive sulfur species, are generally formed during sulfur amino acid metabolism and also occur in a certain protein cysteine residues, including cysteine hydropersulfides (CysSSH), glutathione hydropersulfides (GSSH), and protein cysteine hydropersulfides. The biosynthesis of these species mainly depends on sulfurtransferases, such as cystathionine $\beta$-synthase (CBS) and cystathionine $\gamma$-lyase (CSE) ${ }^{1,2}$ Another important source of RSSH is 3-mercaptopyruvate sulfurtransferase $(\mathrm{MpST})$. $^{2}$ In addition, RSSH can also be produced from other pathways involving enzymes such as cystathionase (CST), cysteine transaminase, and rodanese (thiosulfate: cyanide sulfurtransferase). ${ }^{3,4}$ The various intermediates possess important chemical and biological properties and play crucial roles in signal transduction, redox homeostasis, and metabolic regulation. RSSH dysregulation will lead to unexpected changes in protein function and redox signal regulation and further lead to inflammatory and even disease development, such as cardiovascular disease, Alzheimer's disease, neurodegenerative disease, and even cancers. ${ }^{5}$ Therefore, RSSH is now the focus of intense investigations due to its importance in every aspect of cell biology from the structural function of proteins to the redox signal pathway. ${ }^{6}$
Many metallic elements play important roles in biological systems by covalent modification of protein residues. However, some toxic metallic elements can also affect cell functions by this means, for example divalent mercury ion $\left(\mathrm{Hg}^{2+}\right) . \mathrm{Hg}^{2+}$ is a toxic heavy metal ion which can easily enter organisms. ${ }^{7,8} \mathrm{Hg}^{2+}$ can interfere with normal physiological activities by binding to proteins with physiological regulation function especially those containing the sulfhydryl group. ${ }^{9}$ Research has shown that active $\mathrm{RSSH}$ such as GSSH will compete for $\mathrm{Hg}^{2+}$ with other proteins to form adducts. ${ }^{10}$ Further studies indicate that these adducts are detoxified metabolites of $\mathrm{Hg}^{2+}$. ${ }^{11}$ We believe RSSH with higher nucleophilicity is more competitive than other proteins that possess a sulfhydryl group to form mercury ion adducts. How RSSH protects organisms from mercury poison is not clear. Given the complex relationship between RSSH and $\mathrm{Hg}^{2+}$, effective tools are urgently required to explore this process.

Received: March 26, 2019

Accepted: May 24, 2019

Published: May 24, 2019 

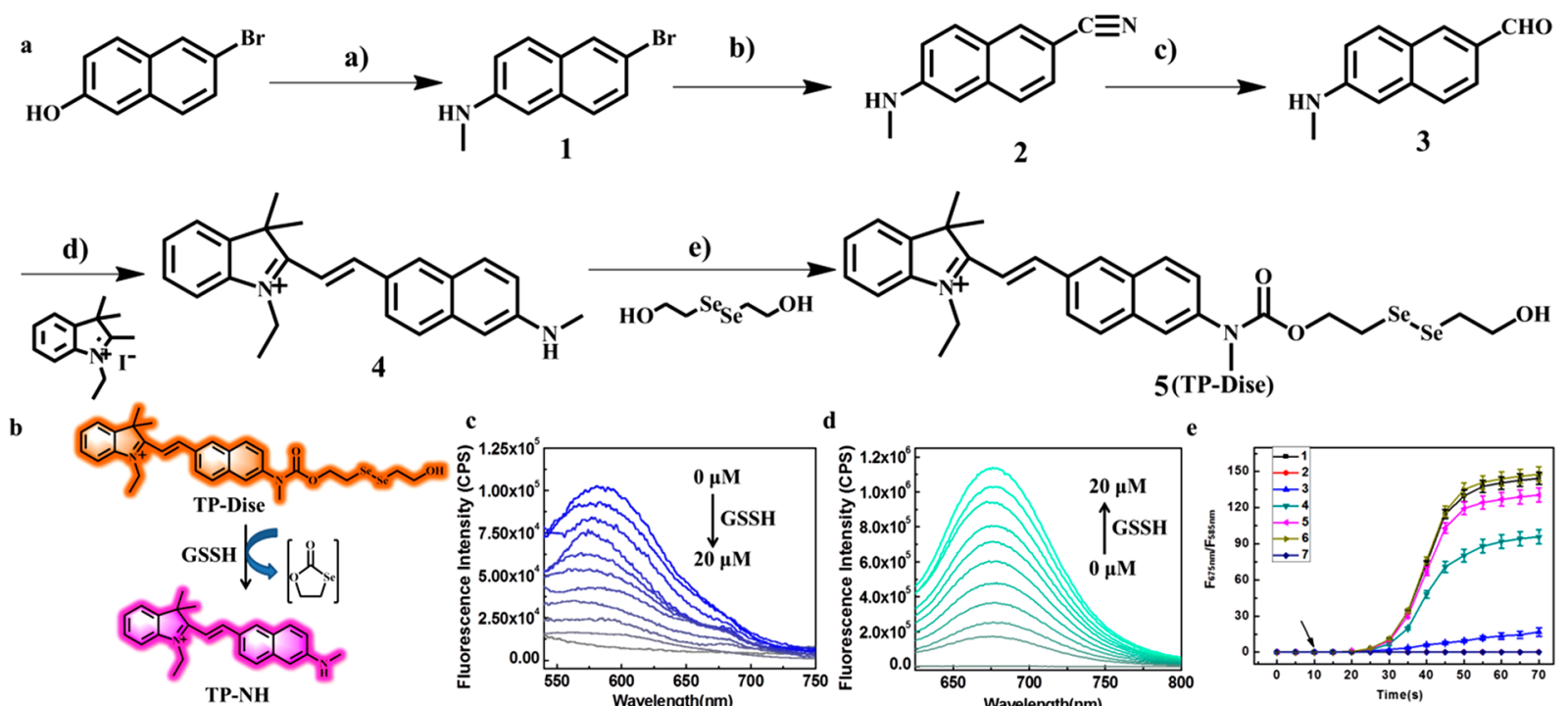

Figure 1. (a) Synthesis route of TP-Dise. (b) Proposed mechanism for GSSH detection. (c) From top to bottom: TP-DiSe (10 $\mu \mathrm{M})$ toward 0, 2, 4, 6, 8, 10, 12, 14, 16, 18, $20 \mu \mathrm{M}$ GSSH, with $\lambda_{\text {ex }}=440 \mathrm{~nm}$. (d) From bottom to top: TP-DiSe $(10 \mu \mathrm{M})$ toward 0, 2, 4, 6, 8, 10, 12, 14, 16, 18, 20 $\mu \mathrm{M}$ GSSH, with $\lambda_{\mathrm{ex}}=560 \mathrm{~nm}$. (e) Time-dependent fluorescent spectra of TP-Dise $(10 \mu \mathrm{M})$ toward GSSH, GSH, and persulfide-BSA in Tris-HCl buffer solution. (1) $20 \mu \mathrm{M}$ GSSH; (2) $20 \mu \mathrm{M} \mathrm{GSH}$; (3) BSA (20 $\mu \mathrm{M}$ ); (4) $20 \mu \mathrm{M} \mathrm{BSA,} 20 \mu \mathrm{M} \mathrm{NaBH}_{4}$; (5) $20 \mu \mathrm{M} \mathrm{BSA}, 30 \mu \mathrm{M} \mathrm{NaBH}$; (6) 20 $\mu \mathrm{M}$ BSA, $40 \mu \mathrm{M} \mathrm{NaBH}_{4}$; (7) $40 \mu \mathrm{M} \mathrm{NaBH}_{4}$, and then these above solutions were treated with NaHS and P-NONOate in Tris-HCl buffer solution to obtain persulfide products. Line 2 and line 7 coincided.

Traditional methods for RSSH detection are based on 2,4dinitrothiophenol methods, modified biotin, ferric thiocyanate, tag-switch assays, ion chromatography, and gas chromatography. ${ }^{12-14}$ However, these methods require complicated sample pretreatment and destruct tissue or cells so that they cannot achieve real-time and in situ imaging. Fluorescent probe technology has been seen as a powerful tool for reactive species detection in vivo owing to its good sensitivity, highly selectivity, less invasiveness, rapid response time, high spatial, and temporal resolution. ${ }^{15}$ Thus, we strive to design a high selective and sensitive fluorescent probe for RSSH detection.

Herein, we developed a two-photon ratiometric fluorescent probe (TP-Dise) based on a new two-photon fluorophore for GSSH detection under mercury stress for the first time. The probe consisted of a new two-photon fluorophore and a testing unit, reactive diselenide (R-Se-Se-R), as GSSH response modulator. The probe could be used for GSSH imaging as well as evaluating the concentration fluctuation of GSSH in living cells, and the results were further confirmed by flow cytometry analysis. In the cell experiments we found that $\mathrm{Hg}^{2+}$ caused a decrease in GSSH concentration. Furthermore, we found that $\mathrm{Hg}^{2+}$ led to cell damage by causing mitochondrial autophagy. GSSH as an antagonist and antioxidant of $\mathrm{Hg}^{2+}$ could alleviate the damage caused by mercury stress. TP-Dise was further applied for GSSH imaging in brain tissue and the imaging depth of TP-Dise up to $100 \mu \mathrm{m}$.

\section{EXPERIMENTAL SECTION}

Synthesis of Compound 4. Hemicyanine ( $1 \mathrm{~g}, 3.17$ mmol) and compound $3(0.5 \mathrm{~g}, 2.7 \mathrm{mmol})$ were dissolved in $n$ butyl alcohol and benzene $(7: 3 \mathrm{v} / \mathrm{v})$ and refluxed for $4 \mathrm{~h}$. After concentration, the obtained crude product was purified by silica column chromatography (200-300 mesh) to afford a fuchsia product $(0.75 \mathrm{~g}, 78 \%) .{ }^{1} \mathrm{H}$ NMR (500 MHz, DMSO$\left.d_{6}\right) \delta(\mathrm{ppm}): 1.47(\mathrm{t}, 3 \mathrm{H}), 1.83(\mathrm{~s}, 6 \mathrm{H}), 2.850-2.990(\mathrm{~d}, 3 \mathrm{H})$, 4.640-4.760 (q, 2H), $6.800(\mathrm{~d}, 1 \mathrm{H}), 6.890-6.970(\mathrm{q}, 1 \mathrm{H})$, 7.040-7.110 (d, $1 \mathrm{H}), 7.515-7.690(\mathrm{~m}, 3 \mathrm{H}), 7.695-7.795(\mathrm{q}$,
$2 \mathrm{H}), 7.835-7.915$ (d, 2H), 8.175-8.250 (d, 1H), 8.495-8.600 (d, 2H). LC-MS (API-ES): $m / z \quad \mathrm{C}_{25} \mathrm{H}_{27} \mathrm{~N}_{2}{ }^{+}$Calcd 355.22, found $\left[\mathrm{M}^{+}\right] 355.38$.

Synthesis of Compound 5 (TP-Dise). Compound 4 $(0.0355 \mathrm{~g}, 0.1 \mathrm{mmol})$ and triphosgene $(0.09 \mathrm{~g}, 0.3 \mathrm{mmol})$ were dissolved in $50 \mathrm{~mL}$ of anhydrous dichloromethane under $\mathrm{Ar}$ atmosphere, and then $1 \mathrm{~mL}$ of DIPEA was added dropwise. The mixture was reacted at $0{ }^{\circ} \mathrm{C}$ for $30 \mathrm{~min}$. After solvent removal in vacuum, the obtained residue was dissolved in 50 $\mathrm{mL}$ of anhydrous $\mathrm{CH}_{2} \mathrm{Cl}_{2}$, and DIPEA ( $\left.1 \mathrm{~mL}\right)$ and DMAP (20 $\mathrm{mg}$ ) were added. Bis(2-hydroxyethyl) diselenide (0.050 g, 0.2 $\mathrm{mmol})$ in $\mathrm{CH}_{2} \mathrm{Cl}_{2}(2 \mathrm{~mL})$ was added into the above mixture, the reaction mixture was stirred at $25{ }^{\circ} \mathrm{C}$, and TLC was monitored until the starting material was completely consumed. The obtained solid residue was purified through a silica gel column (200-300 mesh) with a gradient eluent of $\mathrm{CH}_{2} \mathrm{Cl}_{2}$ and $\mathrm{CH}_{3} \mathrm{OH}(30: 0$ to $15: 1 \mathrm{v} / \mathrm{v})(21 \mathrm{mg}$, yield 33\%). ${ }^{1} \mathrm{H}$ NMR (500 MHz, CD $\left.{ }_{3} \mathrm{OD}-d\right) \delta(\mathrm{ppm}): 0.97(\mathrm{t}, 6 \mathrm{H}), 1.71$ $(\mathrm{t}, 3 \mathrm{H}), 2.01(\mathrm{t}, 2 \mathrm{H}), 2.15-2.18(\mathrm{~m}, 2 \mathrm{H}), 2.90(\mathrm{~s}, 1 \mathrm{H}), 3.39$ $(\mathrm{s}, 3 \mathrm{H}), 3.43(\mathrm{~s}, 2 \mathrm{H}), 3.56(\mathrm{~m}, 2 \mathrm{H}), 3.66(\mathrm{~m}, 2 \mathrm{H}), 5.48-5.50$ $(\mathrm{d}, 2 \mathrm{H}), 6.00-6.04(\mathrm{~d}, 2 \mathrm{H}), 7.60(\mathrm{~m}, 3 \mathrm{H}), 7.70(\mathrm{~m}, 3 \mathrm{H}), 7.87$ (d, 2H), 7.94-7.96 (q, 1H), 8.07-8.09 (d, 2H). LC-MS $\left(\mathrm{ESI}^{+}\right): \quad m / z \quad \mathrm{C}_{30} \mathrm{H}_{35} \mathrm{~N}_{2} \mathrm{O}_{3} \mathrm{Se}_{2}^{+}$calcd. 629.53, found $\left[\mathrm{M}^{+}\right]$ 629.13.

Synthesis. Other compounds were synthesized according to the general procedure, and characterization details are described in Supporting Information.

Laser Confocal Imaging. The cell images in Figure 2 and Figure 3 were acquired using an Olympus Fluo View FV1000 confocal laser-scanning microscope (Japan). The fluorescence signals were collected from 540 to $640 \mathrm{~nm}\left(\lambda_{\mathrm{ex}}=440 \mathrm{~nm}\right.$, channel 1) and from 650 to $750 \mathrm{~nm}\left(\lambda_{\mathrm{ex}}=535 \mathrm{~nm}\right.$, channel 2).

Established Mice Model of Mercury Stress. BALB/c mice were obtained from Binzhou Medical University. Mice weighing about $20 \mathrm{~g}$ were group-housed on a SPF laboratory diet, and water was acquired freely. Model mice: $18 \mathrm{mg} / \mathrm{kg}$ mercury chloride solution was given to mice orally by gavage 
$(0.2 \mathrm{~mL})$ for 3 days, and the control mice were given equivalent saline.

\section{RESULTS AND DISCUSSION}

Design and Synthesis of Probe. Fluorescent probes exhibit excellent performance in exploring the biological function of endogenous reactive species in live cells, but only several fluorescent probes are currently available for RSSH detection. On the basis of nucleophilic attack, the Y. Urano group and A. Ojida group developed two one-photon fluorescent probes for RSSH detection, respectively. ${ }^{15,16}$ In addition, we reported a fluorescent probe for RSSH detection based on selenium-sulfur exchange reaction. ${ }^{17}$ The popular probes for hydropersulfides are merely one-photon fluorescence imaging, which is limited to cell imaging and is not useful for deep-tissue imaging. Moreover, there has been no research on the roles of RSSH under exposure to environmental factors. Two-photon fluorescent probes that absorb two near-infrared (NIR) photons have unique advantages such as deeper tissue imaging, longer observation time, less tissue damage, less autofluorescence interference, and higher spatiotemporal resolution. ${ }^{18,19}$ Previous studies have shown that the concentration of GSSH is much greater than that of Cys-SSH; thus, GSSH is the main component of RSSH. ${ }^{1,38}$ We selected GSSH to mimic RSSH in the following studies. Herein we aimed at a two-photon fluorescent probe for GSSH detection. Inspired by two compounds, Flu 1 and hCy-OH (Scheme S1), we synthesized a new fluorophore, TP-NH (compound 4, Figure 1a) with two-photon character. ${ }^{20,21}$ The new fluorophore emits NIR fluorescence at $675 \mathrm{~nm}$ which has a longer red shift, about $75 \mathrm{~nm}$ compared to that of $\mathrm{hCy}-\mathrm{OH}$. Then we selected the diselenide (R-Se-Se-R) group as the receptor, because of its high reactivity toward GSSH and lack of interference from biothiols, which was adopted in our previous studies. ${ }^{17}$ The probe TP-Dise was synthesized according to Figure 1a. All the compounds were fully characterized and are included in Supporting Information. The response modulator was linked to the nitrogen atom of the fluorophore through an electron-withdrawing carbonyl group. After modification by the response modulator, the electron cloud density on $\mathrm{N}$ shifts to the carbonyl, creating a more electron-deficient substituent as well as resulting in ICTinduced blue shifts in emission maxima. Once the diselenium bond is broken by GSSH, the dye platform performs a rapid intramolecular cyclization back to a methylamino group, resulting in more effective internal charge transfer (ICT), as well as a spectral red shift (Figure $1 \mathrm{~b}$ ).

Spectroscopic Properties of the Probe toward GSSH. The spectroscopic properties of TP-Dise were explored under simulated physiological conditions. The absorption spectra of TP-Dise were investigated in DMSO, ethanol, and Tris- $\mathrm{HCl}$ buffer (Figure S1). We found that the probe had almost no spectral differences in different solvents; thus, we selected Tris$\mathrm{HCl}$ buffer (Tris- $\mathrm{HCl}$ buffer, $\mathrm{pH}$ 7.4) as the buffer system for further study. UV-vis spectra of TP-Dise showed absorption band maxima at $433 \mathrm{~nm}$ (Figure S1). After treatment of TPDise with GSSH, a new absorption peak appeared at $552 \mathrm{~nm}$. Then the fluorescence spectra of TP-Dise were further investigated. The probe had emission maxima at $585 \mathrm{~nm}$ (Figure 1c). Upon reaction with GSSH, the maximum emission of the probe changed from 585 to $675 \mathrm{~nm}$ which was located in the NIR region (Figure 1d). Furthermore, the ratio $\left(F_{675 \mathrm{~nm}} / F_{585 \mathrm{~nm}}\right)$ was positively correlated with GSSH
(Figure S2a) and showed a linear relationship with the concentration of GSSH $(0-20 \mu \mathrm{M}$, Figure S2b). The regression equation between $\lg \left(F_{675 \mathrm{~nm}} / F_{585 \mathrm{~nm}}\right)$ and the concentrations of GSSH was $\lg \left(F_{675 \mathrm{~nm}} / F_{585 \mathrm{~nm}}\right)=0.09746$ [GSSH] $\mu \mathrm{M}+0.08613$, with $r=0.9980$. The detection limit was determined to be $89 \mathrm{nM}(3 \sigma / k)$.

Then the selectivity of the probe was investigated carefully. The $\mathrm{p} K_{\mathrm{a}}$ of glutathione (GSH), a main thiol at millimolar level, is 8.75. ${ }^{22}$ However, the $\mathrm{p} K_{\mathrm{a}}$ of GSSH is about 7.0 which shows stronger acidity compared to $\mathrm{GSH}^{23}$ At physiological $\mathrm{pH}$ (7.40), most biothiols exist as the protonated forms, while GSSH primarily exists as the deprotonated anion $\left(\mathrm{GSS}^{-}\right)$ which is a stronger nucleophilic reagent than protonated biothiols. Thus, reactive sulfur species (RSS) such as cystine (Cys-S-S-Cys), GSH, and oxidized glutathione (GSSG) will not interfere with GSSH detection. Then we simulated the physiological environment in the organisms to performed selectivity experiments (Figure S4b). We used $20 \mu \mathrm{M}$ GSSH, and $4 \mu \mathrm{M}$ Cys-SSH, one-fifth the concentration of GSSH, for further research. As shown in Figure S4b, GSSH caused fluorescence signals stronger than that of Cys-SSH under simulated physiological condition. More detailed selectivity tests are shown in Figure S4a. Other interferences such as Hcy, GSH, GSSG, $\mathrm{Fe}^{3+}, \mathrm{Zn}^{2+}, \mathrm{Ca}^{2+}$, etc., could not interfere with GSSH detection. Furthermore, we tested persulfide P, an analogue of the nitrosothiol $S$-nitrosoacetylpenicillamine (SNAP), to verify the detection (Figure S4a). In addition, we used BSA (albumin from bovine serum) to mimic macromolecular thiol proteins for GSSH monitoring. BSA is a kind of globulin that contains 17 disulfide bonds composed of 35 cysteines. We used $\mathrm{NaBH}_{4}$ to break disulfide bonds to get a greater number of sulfhydryl groups. After the formation of GSSH, we performed spectral tests. ${ }^{1}$ As shown in Figure 1e, bright fluorescence signals indicated that our probe was a promising tool for intracellular fluorescence imaging. To demonstrate that the fluorescent enhancement was the result of the generation of persulfide-BSA, we performed an additional experiment. As shown in Figure 1e line 7, the fluorescence changes were very small. These results demonstrated that the fluorescent enhancement was the result of the generation of persulfide-BSA. In addition, the reaction kinetic of TP-Dise was performed in Tris- $\mathrm{HCl}$ buffer. The saturated fluorescence intensity could be observed after incubation of GSSH within $60 \mathrm{~s}$ (Figure S3). We speculated that positively charged hemicyanine had a great impact on the nucleophilic reaction rate. Thus, our probe could response to GSSH and simulated macromolecular protein hydropersulfides which provided the basis for intracellular imaging. In addition, we detected the probe stability under different $\mathrm{pH}$ values (Figure S5). The results demonstrated that our probe was stable enough for GSSH detection.

GSSH Detection in Living Cells. On the basis of the good spectral response of TP-Dise, we applied TP-Dise as a promising tool for GSSH detection in living cells. Before cell imaging, MTT assays were performed to evaluate cell cytotoxicity of TP-Dise (Figure S6). Different cell lines displayed high cell activity which indicated that TP-Dise had low cytotoxicity to living cells. Then we selected SH-SY5Y cells and A549 cells as biological models to research the concentration fluctuations and biosynthesis of GSSH. The time-dependent ratio fluorescence intensities of GSSH were detected in 0-105 s (Figure 2A). Fluorescence intensity increased over time, and different rates of change demon- 


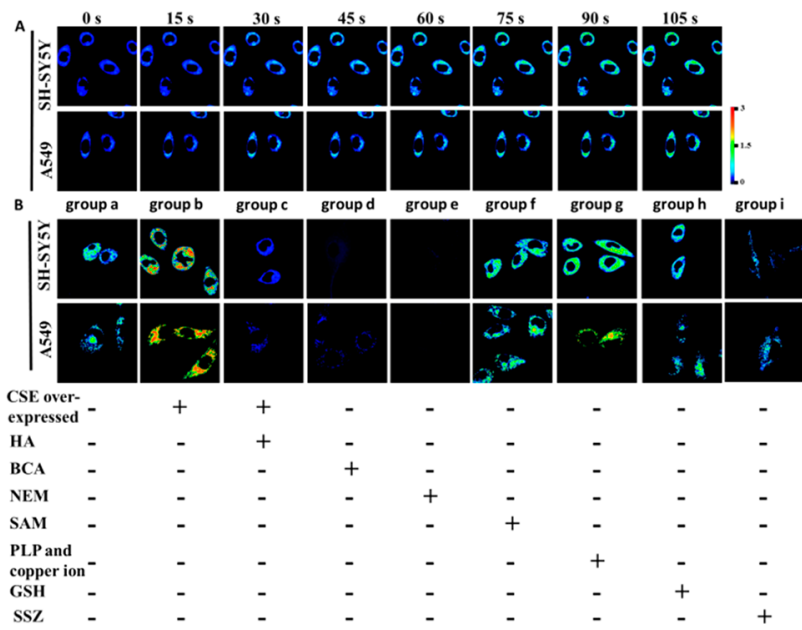

Figure 2. (A) Time-dependent ratio images (Fchannel 2/Fchannel 1) of endogenous GSSH analyses in SH-SY5Y cells and A549 cells. (B) Ratio images of GSSH generation in living SH-SY5Y cells and A549 cells. Group a: $10 \mu \mathrm{M}$ TP-Dise; group b: CSE-overexpressed cells; group c: $250 \mu \mathrm{M}$ HA; group d: $50 \mu \mathrm{M}$ BCA; group e: $5 \mathrm{mM}$ NEM; group f: $3 \mathrm{mM}$ SAM; group g: $1 \mu \mathrm{M}$ PLP and $1 \mu \mathrm{M}$ copper ion; group h: $100 \mu \mathrm{M} \mathrm{GSH}$; group i: $100 \mu \mathrm{M}$ SSZ.

strated the different cell lines' ability to produce endogenous GSSH. Then we selected the time point at $105 \mathrm{~s}$ for GSSH detection in SH-SY5Y cells and A549cells. Our probe could response to GSSH in cells without other interferences,.

Next, we investigated the biosynthetic pathway of GSSH in living cells (Figure 2B). Cells in group a were incubated with TP-Dise $(10 \mu \mathrm{M})$ only. A bright ratiometric fluorescence signal could be observed, and the results indicated that a considerable amount of GSSH existed in cells for redox balance. The enzyme CSE in group $b$ was overexpressed. ${ }^{1}$ The overexpressed plasmid was encapsulated into liposomes by Lipofectamine 2000 (Invitrogen) and then coincubated with the cells to obtain CSE-overexpressed cells. A stronger ratiometric fluorescence signal could be observed. Hydroxylamine (HA) is an inorganic compound $\left(\mathrm{NH}_{2} \mathrm{OH}\right)$, and it can be used as an inhibitor for CBS and CSE. ${ }^{24,25}$ Then HA was used to inhibit enzyme activity in CSE-overexpressed cells. A low ratiometric fluorescence signal in group $\mathrm{c}$ indicated that the concentration of GSSH was low. $\beta$-Cyanoalanine (BCA) is a reversible inhibitor of CSE and works by modifying the apoenzyme transiently. ${ }^{25,26}$ Then cells in group $d$ were incubated with BCA, and the cells showed weak ratiometric fluorescent signal indicated that GSSH production was clearly inhibited. $N$ Ethylmaleimide (NEM) is a thiol scavenger to exhaust endogenous RSS. Cells in group e were treated with NEM for $30 \mathrm{~min}$. S-Adenosyl-L-methionine (SAM) is a key intracellular regulator, and it can serve as a typical allosteric enzyme activator to promote RSS generation. ${ }^{27}$ In group $f$, cells were incubated with SAM to facilitate GSSH production. RSS can be generated from the nonenzymic $\alpha, \beta$-elimination reaction. The concentration of GSSH increased upon catalysis by pyridoxal phosphate (PLP) and copper ion. ${ }^{28}$ Then the cells in group $g$ were pretreated with PLP and copper ion. A brighter ratiometric fluorescence signal could be collected. Thus, PLP and copper ion could be used for GSSH production. The cells in group $h$ were incubated with GSH $(100 \mu \mathrm{M})$ for $1 \mathrm{~h}$, and the concentration of GSSH increased. Cystine/glutamine transporter $(\mathrm{xCT})$ is a major amino acid transporter and responsible for the intracellular supply of CysSSCys which is functionally linked to GSSH biosynthesis. ${ }^{1}$ Sulfasalazine (SSZ) is a potent inhibitor of $\mathrm{xCT} .{ }^{29}$ Then cells in group i were treated with SSZ for $3 \mathrm{~h}$, and decreased ratiometric fluorescence intensity could be observed. Comparing the fluorescence intensity in Figure 2B, we found that the main biological sources of GSSH were two sulfurtransferases: CBS and CSE. The fluorescence ratios of each group were analyzed in Figure S7b and S7c. In addition, flow cytometry analysis is a kind of high-throughput assay technology that benefits from its high precision for abundant sample analysis. The GSSH detections from laser scanning confocal microscopy were confirmed by flow cytometry analysis (Figure S7a). Their ratio analyses are shown in Figure S7d and S7e. Thus, our probe was a powerful chemical tool for observation of the biosynthesis of GSSH.

Evaluation of the Biological Protection Functions of GSSH. Then we explored the biological protection function of GSSH on mercury-stressed organisms. As shown in Figure 3, cells were first incubated with $10 \mu \mathrm{M}$ probe for $5 \mathrm{~min}$ as control, and we could observe bright ratiometric fluorescence signals. The results indicated that cells maintained normal redox balance in a cell microenvironment. The cells in other groups were manipulated as the control group. Next the cells were pretreated with $10 \mu \mathrm{M} \mathrm{Hg}^{2+}$ for $12 \mathrm{~h}$. Weak ratiometric

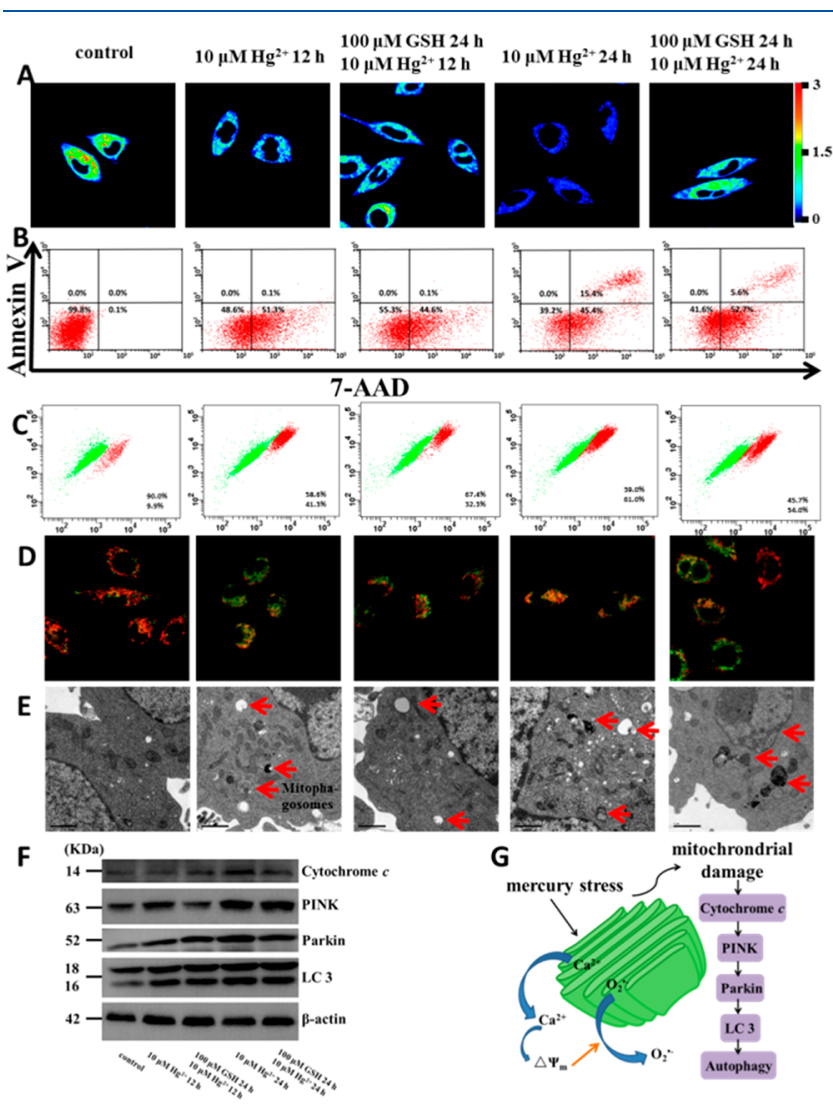

Figure 3. (A) Confocal microscope imaging. (B) Apoptosis analysis: viable cells (AnnexinV-/7-AAD-), early apoptosis (Annexin V+/7$\mathrm{AAD}-$ ), late apoptosis (AnnexinV+/7-AAD+), necrosis (AnnexinV-/ 7-AAD+). (C) $\Delta \Psi \mathrm{m}$ analyzed by JC-1. (D) Merged picture of mitochondrial imaging and lysosomal imaging. (E) TEM imaging (scale bars: $1 \mu \mathrm{m}$ ). (F) Western blot analysis for cytochrome $c$, PINK, Parkin, and LC3 analysis. (G) Schematic mechanism of GSSH for protecting cells from mercury stress. 


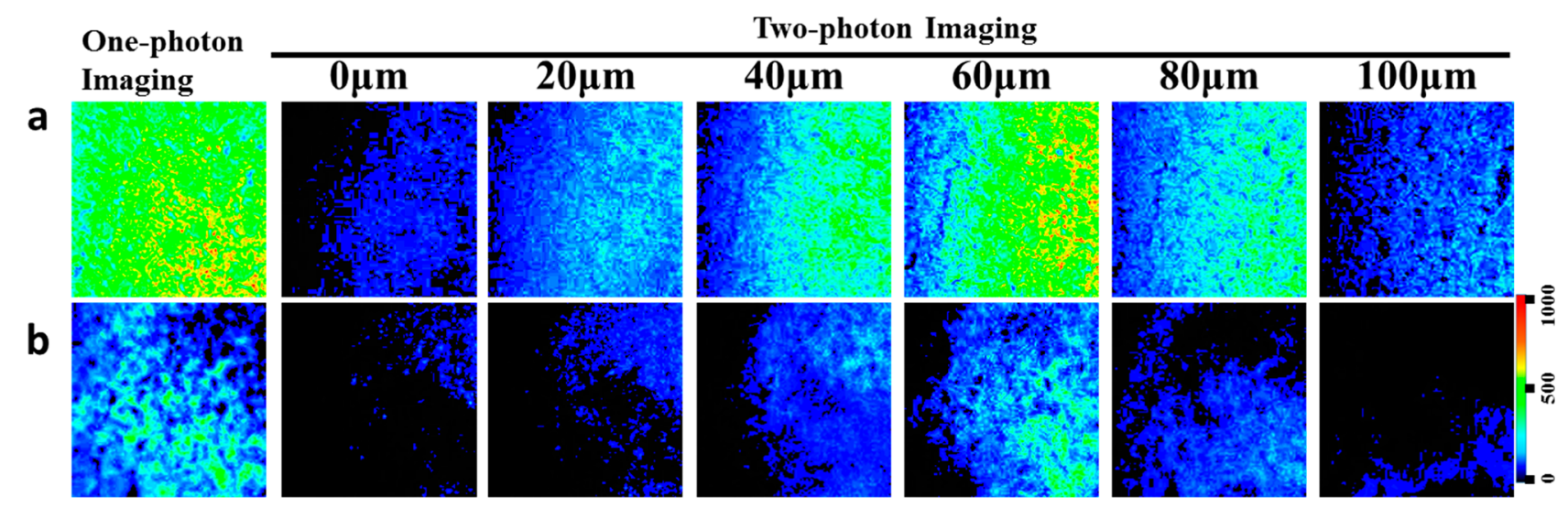

Figure 4. GSSH detection in brain slices. (a) Brain slices from the mice in control group. (b) Brain slices from the mice in the model group. Twophoton imaging: channel $1, \lambda_{\mathrm{ex}}=880 \mathrm{~nm}, \lambda_{\mathrm{em}}=540-640 \mathrm{~nm}$; channel $2, \lambda_{\mathrm{ex}}=1080 \mathrm{~nm}, \lambda_{\mathrm{em}}=650-750 \mathrm{~nm}$.

fluorescence signals could be observed, which revealed that the concentration of GSSH decreased. Then the cells were incubated with $100 \mu \mathrm{M}$ GSH for $24 \mathrm{~h}$ before addition of $\mathrm{Hg}^{2+}$. The intensity of ratiometric fluorescence signal was increased. The results demonstrated that GSH could effectively increase the GSSH concentration to protect cells from damages. After that, the cells were incubated with $10 \mu \mathrm{M}$ $\mathrm{Hg}^{2+}$ for $24 \mathrm{~h}$. Compared to the ratiometric fluorescence signal in the second group, the lower ratiometric fluorescence signals in the fourth group were detected. We considered that much more GSSH was consumed by $\mathrm{Hg}^{2+}$ for detoxification. Then the cells in the fifth group were pretreated with $100 \mu \mathrm{M} \mathrm{GSH}$ for $24 \mathrm{~h}$ before addition of $\mathrm{Hg}^{2+}$. Compared to the fourth group, a higher ratiometric fluorescence intensity could be observed in the fifth group. The corresponding fluorescence quantitative analyses are shown in Figure S10b. The results from confocal microscopy were further confirmed by flow cytometry analysis (Figure S10a), and the quantitative analyses are shown in Figure S10c.

The cell apoptosis analyses of each group were performed by Annexin V/7-AAD Apoptosis Detection Kit (Figure 3B). As a cytotoxin, $\mathrm{Hg}^{2+}$ definitely causes cell damage. We used apoptotic rate (including necrosis) to assess the degree of cell damage which could be sorted as fourth group $>$ fifth group $>$ second group $>$ third group $>$ first group. Intracellular imbalance could induce a series of anomalous events such as mitochondrial membrane potential $(\Delta \Psi \mathrm{m})$ decrease accompanied by opening of the mitochondrial permeability transition pore. ${ }^{30}$ We next detected $\Delta \Psi \mathrm{m}$ by JC-1 to confirm apoptotic damage (Figure $3 \mathrm{C}$ ). The decrease in green-red fluorescence ratio represented a reduction in cell mitochondrial membrane potential and aggravation of cell damage. The degree of cell damage could be sorted as fourth group $>$ fifth group $>$ second group $>$ third group $>$ first group. These results demonstrated that cell damage was aggravated with the prolongation of mercury stress as well as the increase in $\mathrm{Hg}^{2+}$ concentration. Through cell damage analysis, we found that GSSH could protect cells from mercury stress. We deduced that GSSH can release mercury poison in two ways. First, the highly nucleophilic GSSH as an antagonist of $\mathrm{Hg}^{2+}$ is more competitive to form mercury complexes for detoxification. Second, GSSH can serve as a stronger antioxidant to eliminate the outbreak of reactive oxygen species caused by $\mathrm{Hg}^{2+}$.

Research has indicated that mercury stress can induce mitochondrial autophagy, and we explore the process through fluorescence imaging, transmission electron microscopy (TEM), and Western blot. ${ }^{31}$ Mitochondrial autophagy is a regulated process in which mitochondria are surrounded by a bilayer membrane structure and fuse with lysosomes ultimately for degradation. ${ }^{32,33}$ Thus, we preliminarily studied the process of mitochondrial autophagy using a mitochondrial dye and a lysosomal dye (Figure 3D). ${ }^{34}$ Red stands for lysosomal dye, green stands for mitochondrial dye, and yellow stands for the coincident part of the two dyes. We found that as deterioration occurs with cell damage, the coincidence of mitochondrial and lysosomal dyes increased. According to the experimental results, we preliminarily speculated that the mitochondria had autophagy. Then we observed the ultrastructure of cells by TEM. As shown in Figure 3E, in the control group (first group) we could find regular mitochondria, clear and continuous nuclear membranes, mitochondria ridges as well as cell membranes. In the experimental groups, we found that mitochondria swelled and deformed, the organelle membrane became blurred, and the number of vacuoles increased. Furthermore, we found mitochondrial autophagosomes in the experiments of the second group. Thus, we believed that mercury stress could cause mitochondrial autophagy and cell damage. Then we performed Western blot tests to explore the process of mitochondrial autophagy. Mercury stress led to mitochondrial membrane potential collapse and further caused cytochrome $c$ in mitochondria to be released into cytoplasm. LC3 is a marker protein for detecting autophagy, ${ }^{36}$ and the proteins were detected. As shown in Figure 3F, obviously protein expression of LC3 in the mercury treatment groups could be observed which demonstrated that $\mathrm{Hg}^{2+}$ induced mitochondrial autophagy. Under normal conditions, the expression of PINK was very low. After mitochondria was damaged, PINK could be stably accumulated and induce Parkin cumulation. ${ }^{37}$ Next, the proteins PINK and Parkin were detected. We found that the protein expression of PINK and Parkin up-regulated with cell damage became much more severe. The expression of cytochrome $c$ was also increased. The results demonstrated that mercury stress induced cell damage and mitochondrial autophagy. The schematic mechanism of GSSH for protecting cells from mercury stress is shown in Figure 3G.

Two-Photon Fluorescent Probe TP-Dise for GSSH Detection in Deep Brain Tissue. Because the two-photon fluorescent probe TP-Dise could achieve cell imaging at different depths successfully in Figure S13, we further 
performed GSSH detection in brain tissue for deep tissue imaging. Then we constructed a mice model of mercury stress, and the GSSH fluctuation in the brain was detected by TPDise (Figure 4). The mice were divided into two groups. Mice in group a were the control group (gastric dose: $0.2 \mathrm{~mL}$ saline for 3 days). Mice in the model group were given mercury chloride $(18 \mathrm{mg} / \mathrm{kg}$ in saline/day, $0.2 \mathrm{~mL}$ per day) orally by gavage for 3 days. The brains from mice were dissected as quickly as possible, and then fresh tissue sections were obtained for quick detection through one-photon microscopy (OPM) and two-photon microscopy (TPM). The fresh tissue sections were stained with $10 \mu \mathrm{M}$ TP-Dise for $10 \mathrm{~min}$. The fluorescence signal could be collected by OPM and TPM. As shown in Figure 4a, we could observe strong fluorescence by OPM. Then the TPM imaging was performed and the imaging depth up to $100 \mu \mathrm{m}$. We could clearly capture the fluorescent signal of each layer to obtain more cell information. Next, the brain tissue sections from the model mice were stained with TP-Dise for imaging. Compared to Figure $4 a$, the fluorescence in Figure $4 \mathrm{~b}$ decreased obviously which represented the concentration of GSSH decreased. To confirm the interference of autofluorescence from tissues, we performed control experiments without dye incubation. As shown in Figure S16, ignorable ratiometric fluorescence signals could be observed. Thus, autofluorescence from tissues did not interfere with the detection of GSSH. The fluorescence imaging results showed that $\mathrm{Hg}^{2+}$ entered brain tissue with blood circulation and decreased the concentration of GSSH. We thought that could be ascribed to two reasons: GSSH reacted with $\mathrm{Hg}^{2+}$, forming GSSH-Hg adducts to decrease GSSH; moreover, the reactive oxygen species burst induced by $\mathrm{Hg}^{2+}$ further decreased the reducing species GSSH. ${ }^{35}$ The noninvasive imaging, rapid response time, and real-time and in situ imaging made our probe a useful chemical tool for GSSH detection in deep tissue.

$\mathrm{Hg}^{2+}$ could cause GSSH decreased as well as tissue damage after entering the brain. Then we evaluated the brain tissue damage through TUNEL and H\&E (hematoxylin and eosin) staining. As shown in TUNEL staining (Figure S17), negligible fluorescence could be observed in the control group. However, we could see significantly enhanced fluorescence in the model group. These results demonstrated that the brain tissue damage in the model group was more serious. Next, we used more intuitive $\mathrm{H} \& \mathrm{E}$ to analyze brain tissue damage. As shown in Figure S18, the brain tissue in the control group shaped rule and arranged closely, while the brain tissue in the model group was loosely arranged and the nucleus appeared deformed. These results further indicated that $\mathrm{Hg}^{2+}$ induced severe tissue damage.

\section{CONCLUSION}

We have designed and synthesized the first two-photon ratiometric fluorescent probe (TP-Dise) for GSSH detection. The probe is used for studying the biosynthesis of GSSH, and we find that GSSH is mainly from two sulfurtransferases, CBS and CSE. Then we explored the roles of GSSH in protecting cells from mercury stress-induced mitochondrial autophagy and damages for the first time. The results of the apoptosis experiments demonstrate that GSSH can alleviate the damage caused by mercury stress and protect cells from two aspects. On the one hand, GSSH as an antagonist can be directly used for $\mathrm{Hg}^{2+}$ detoxification by forming mercury ion complexes. On the other hand, GSSH can be used as an antioxidant for maintaining intracellular redox balance by eliminating the outbreak of ROS caused by $\mathrm{Hg}^{2+}$. The imaging depth of TPDise up to $100 \mu \mathrm{m}$ will capture more information in various organisms and facilitate deep tissue research. In summary, the TP-Dise probe is a powerful candidate for accurate diagnosis and efficacy evaluation physiological pathology of GSSH, which will promote our understanding of biofunctions about RSSH.

\section{ASSOCIATED CONTENT}

\section{Supporting Information}

The Supporting Information is available free of charge on the ACS Publications website at DOI: 10.1021/acs.analchem.9b01511.

Experimental supplementary methods for chemical synthesis and characteristic of compounds, supplementary experiments, and data (PDF)

\section{AUTHOR INFORMATION}

\section{Corresponding Author}

*E-mail: lxchen@yic.ac.cn.

ORCID

Liangwei Zhang: 0000-0001-5317-0087

Zhe Liu: 0000-0001-5796-4335

Lingxin Chen: 0000-0002-3764-3515

Notes

The authors declare no competing financial interest.

\section{ACKNOWLEDGMENTS}

We thank the National Nature Science Foundation of China (nos. 21575159, 21778026) and the program of Youth Innovation Promotion Association, CAS (grant 2019217).

\section{REFERENCES}

(1) Ida, T.; Sawa, T.; Ihara, H.; Tsuchiya, Y.; Watanabe, Y.; Kumagai, Y.; Yamamoto, M.; et al. Proc. Natl. Acad. Sci. U. S. A. 2014, $111,7606-7611$.

(2) Kimura, Y.; Koike, S.; Shibuya, N.; Lefer, D.; Ogasawara, Y.; Kimura, H. Sci. Rep. 2017, 7, 10459.

(3) Iciek, M.; Wlodek, L. Polym. J. Pharmacol. 2001, 53, 215.

(4) Cooper, A. J. Annu. Rev. Biochem. 1983, 52, 187.

(5) Wang, F.; Feng, C.; Lu, L.; et al. Talanta 2017, 169, 149.

(6) Miranda, K. M.; Wink, D. A. Proc. Natl. Acad. Sci. U. S. A. 2014, $111,7505$.

(7) Kim, S. H.; Sharma, R. P. Toxicol. In Vitro 2003, 17, 385.

(8) Neathery, M. W.; Miller, W. J. J. Dairy Sci. 1975, 58, 1767.

(9) Apaydın, F. G.; Baş, H.; Kalender, S.; Kalender, Y. Environ. Toxicol. Pharmacol. 2016, 41, 219.

(10) Zalups, R. K.; Barfuss, D. W. Toxicol. Appl. Pharmacol. 2002, 182, 234.

(11) Nishida, M.; Kumagai, Y.; Ihara, H.; et al. J. Clin. Biochem. Nutr. 2016, 58, 91.

(12) Park, C. M.; Weerasinghe, L.; Day, J. J.; et al. Mol. BioSyst. 2015, 11, 1775.

(13) Wood, J. L. Methods Enzymol. 1987, 143, 25.

(14) Togawa, T.; Ogawa, M.; Nawata, M.; et al. Chem. Pharm. Bull. 1992, 40, 3000.

(15) Umezawa, K.; Kamiya, M.; Urano, Y. Angew. Chem., Int. Ed. 2018, 57, 9346.

(16) Kawagoe, R.; Takashima, I.; Uchinomiya, S.; Ojida, A. Chem. Sci. 2017, 8, 1134.

(17) Han, X.; Yu, F.; Song, X.; Chen, L. Chem. Sci. 2016, 7, 5098.

(18) Lim, C. S.; Masanta, G.; Kim, H. J.; et al. J. Am. Chem. Soc. 2011, 133, 11132 . 
(19) Pawlicki, M.; Collins, H. A.; Denning, R. G.; Anderson, H. L. Angew. Chem., Int. Ed. 2009, 48, 3244.

(20) Li, L.; Zhang, C. W.; Chen, G. Y. J.; et al. Nat. Commun. 2014, $5,3276$.

(21) Liu, G.; Liu, D.; Han, X.; et al. Talanta 2017, 170, 406.

(22) Cuevasanta, E.; Lange, M.; Bonanata, J.; Coitino, E. L.; FerrerSueta, G.; Filipovic, M. R.; Alvarez, B. J. Biol. Chem. 2015, 290, 26866.

(23) Ueda, T.; Akuta, T.; Kikuchi-Ueda, T.; et al. Protein Expression Purif. 2016, 120, 99.

(24) Wang, R. Physiol. Rev. 2012, 92, 791.

(25) Asimakopoulou, A.; Panopoulos, P.; Chasapis, C. T.; et al. Br. J. Pharmacol. 2013, 169, 922.

(26) Whiteman, M.; Winyard, P. G. Expert Rev. Clin. Pharmacol. 2011, 4, 13.

(27) Módis, K.; Coletta, C.; Asimakopoulou, A.; et al. Nitric Oxide 2014, 41, 146.

(28) Toohey, J. I. Biochem. Cell Biol. 1986, 64, 758.

(29) Gout, P. W.; Buckley, A. R.; Simms, C. R.; Bruchovsky, N. Leukemia 2001, 15, 1633.

(30) Ly, J. D.; Grubb, D. R.; Lawen, A. Apoptosis 2003, 8, 115.

(31) Vergilio, C. S.; Carvalho, C. E. V.; Melo, E. J. T. Toxicol. In Vitro 2015, 29, 63.

(32) Zhang, H.; Bosch-Marce, M.; Shimoda, L. A.; et al. J. Biol. Chem. 2008, 283, 10892.

(33) Lemasters, J. J. Rejuvenation Res. 2005, 8, 3-5.

(34) Baregamian, N.; Song, J.; Bailey, C. E.; et al. Oxid. Med. Cell. Longevity 2009, 2, 297.

(35) Wang, Y.; Gao, M.; Chen, Q.; et al. Anal. Chem. 2018, 90, 9769.

(36) Pellacani, C.; Costa, L. G. Environ. Pollut. 2018, 235, 791.

(37) Wang, B.; Abraham, N.; Gao, G.; Yang, Q. Transl. Neurodegener. 2016, 5, 19.

(38) Akaike, T.; Ida, T.; Wei, F. Y.; Nishida, M.; Kumagai, Y.; Alam, M.; Nishimura, A.; et al. Nat. Commun. 2017, 8, 1177. 